\title{
Protée
}

\section{Images et rituel}

\section{Philippe Langlet}

Volume 30, numéro 1, printemps 2002

Les formes culturelles de la communication

URI : https://id.erudit.org/iderudit/006704ar

DOI : https://doi.org/10.7202/006704ar

Aller au sommaire du numéro

Éditeur(s)

Département des arts et lettres - Université du Québec à Chicoutimi

ISSN

0300-3523 (imprimé)

1708-2307 (numérique)

Découvrir la revue

\section{Citer cet article}

Langlet, P. (2002). Images et rituel. Protée, 30(1), 96-108.

https://doi.org/10.7202/006704ar

\section{Résumé de l'article}

La composition des Tableaux maçonniques repose sur le postulat suivant : une illustration de l'enseignement du Grade auquel ils font référence. Affirmer cela suppose un accord sur le sens des termes employés et sur les notions illustrées. Notre réflexion se fera à partir des Tableaux du deuxième grade, de la Maçonnerie anglaise. Les principes mis en jeu se sont stabilisés vers la fin du $\mathrm{XIX}^{\mathrm{e}}$ siècle et constituent une sorte de standard. Les exemples plus récents reprennent ces principes. Nous étudierons sur quoi repose la matérialité de l’objet, puis ce qui y est représenté. Enfin, nous essaierons de trouver les relations entre les éléments des Tableaux et leur niveau de relation avec le rituel.
Ce document est protégé par la loi sur le droit d'auteur. L'utilisation des services d'Érudit (y compris la reproduction) est assujettie à sa politique d'utilisation que vous pouvez consulter en ligne.

https://apropos.erudit.org/fr/usagers/politique-dutilisation/ 

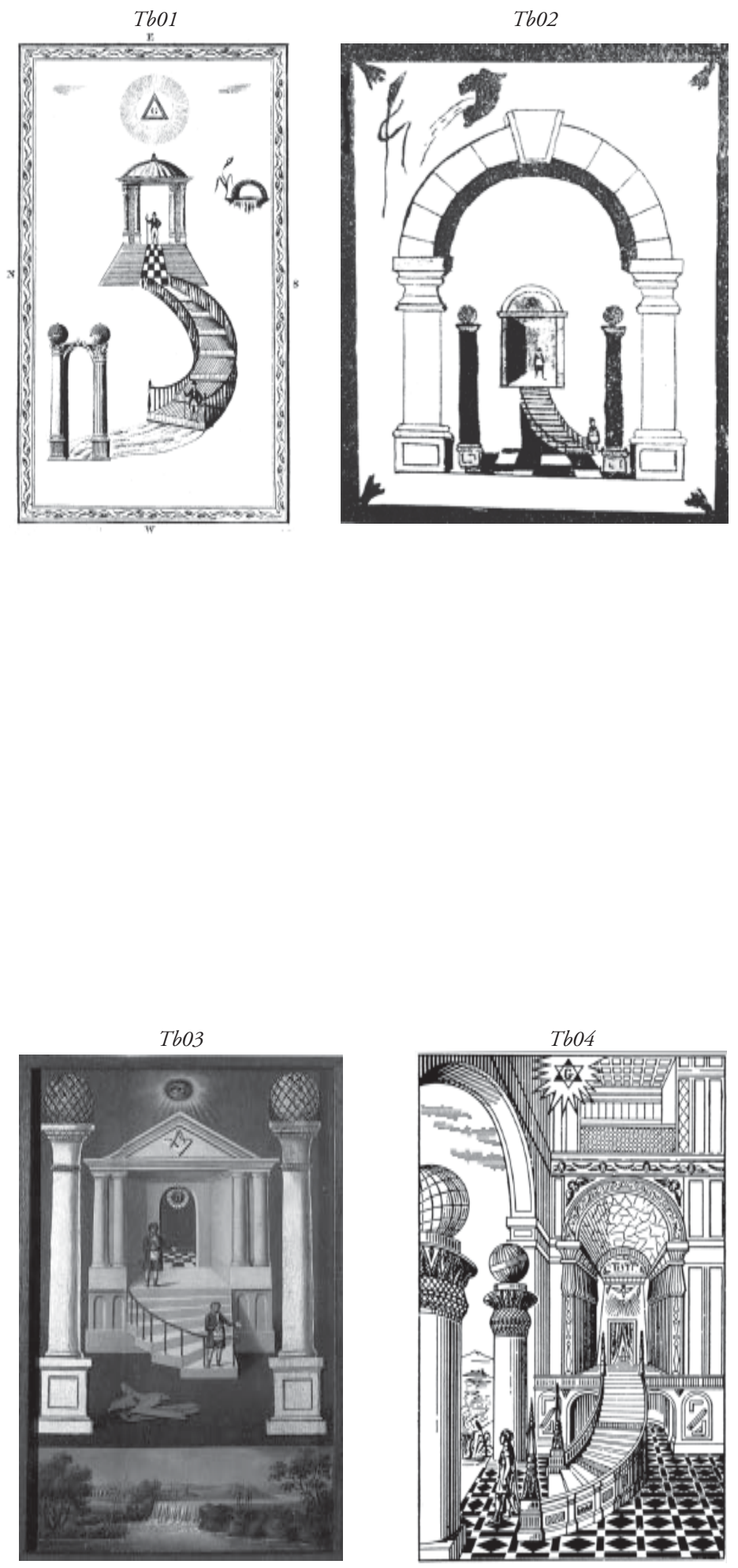


\title{
IMAGES ET RITUEL
}

\author{
PHILIPPE LANGLET
}

Les documents que nous allons étudier sont connus, en France, sous le nom de Tableaux de Loge. Dans les pays de langue anglaise, ce sont des Tracing Boards, terme souvent (mal) traduit en français par «Planches tracées». Nous conserverons le nom de Tableaux de Loge, raccourci par commodité en Tableaux. L'une des caractéristiques de ces images est d'être attachées au système rituel particulier de la Franc-Maçonnerie de métier, ou Maçonnerie symbolique (Craft Masonry ${ }^{1}$ ), qui comporte essentiellement trois grades. Les Tableaux choisis sont rattachés au Deuxième grade. De plus, ils appartiennent à une aire géographique précise, l'Angleterre, et à une période particulière, comprise entre la fin du XVIIIe siècle (c. 1780) et le milieu du XIX ${ }^{\mathrm{e}}$ siècle (c. 1850), quand le rituel se stabilise et où le Tableau de Loge, après quelques avatars, se standardise pour devenir ce qu'il est resté. Le $T b 10$ est une exception: il est récent mais reprend des principes éprouvés. Le premier point que nous évoquerons est la matérialité de l'objet, le second qui retiendra notre attention sera ce qui y est représenté. Le dernier point tentera de trouver les relations entre les éléments des Tableaux.

\section{L'objet}

Le Tableau est une figure plane, à deux dimensions. C'est une portion d'espace, isolé et clôturé par un élément visible, le cadre, à l'intérieur duquel ont été disposés des éléments, en nombre variable, selon une organisation particulière. Le Tableau forme ainsi une unité. Avant de l'étudier en tant qu'unité signifiante, il faut distinguer plusieurs paramètres liés à l'espace qui le comprend: sa forme, son occupation de la surface, son support, le cadre dans lequel il s'inscrit.

Le Tableau est rectangulaire. La caractéristique principale de cette figure, comme du carré, réside dans la coprésence de quatre angles droits et de côtés parallèles deux à deux. Nous conserverons l'appellation de rectangle, bien que la Maçonnerie aime parler de "carré long". Les Tableaux de notre corpus ont tous cette forme, et de manière prononcée, à une exception près (Tb02). Nous dirons que c'est d'abord cette configuration qui définit le Tableau comme tel. Mais ajoutons une précision. Le Tableau est un rectangle posé sur son petit côté, sa longueur étant prise comme hauteur. Son plus grand axe parcourt alors l'espace verticalement (de haut en bas?). Un tableau, au sens artistique, est le plus souvent un rectangle disposé dans l'autre sens, ce qui oriente un balayage visuel, latéral et horizontal, de la surface. Pour une feuille de papier (ou un livre), ce format est «à l'italienne». Le Tableau maçonnique s'inscrit au contraire dans une forme rappelant celle d'une page "ordinaire».

Le Tableau garnit la surface de son support de manière remarquable: il l'occupe toujours dans son intégralité, quelle qu'en soit l'étendue. Il n'existe pas d'exemple de Tableau qui n'occuperait qu'une portion restreinte de la surface. Il s'affirme ainsi comme une manière de la totalité. De plus, le Tableau est centré par rapport à son support. Quelles que soient ses mesures exactes, ses dimensions relatives resteront les mêmes. Il existe déjà une relation entre espace et forme, en existe-t-il une entre forme et support? Quelques Tableaux ont été, à l'origine, réalisés 

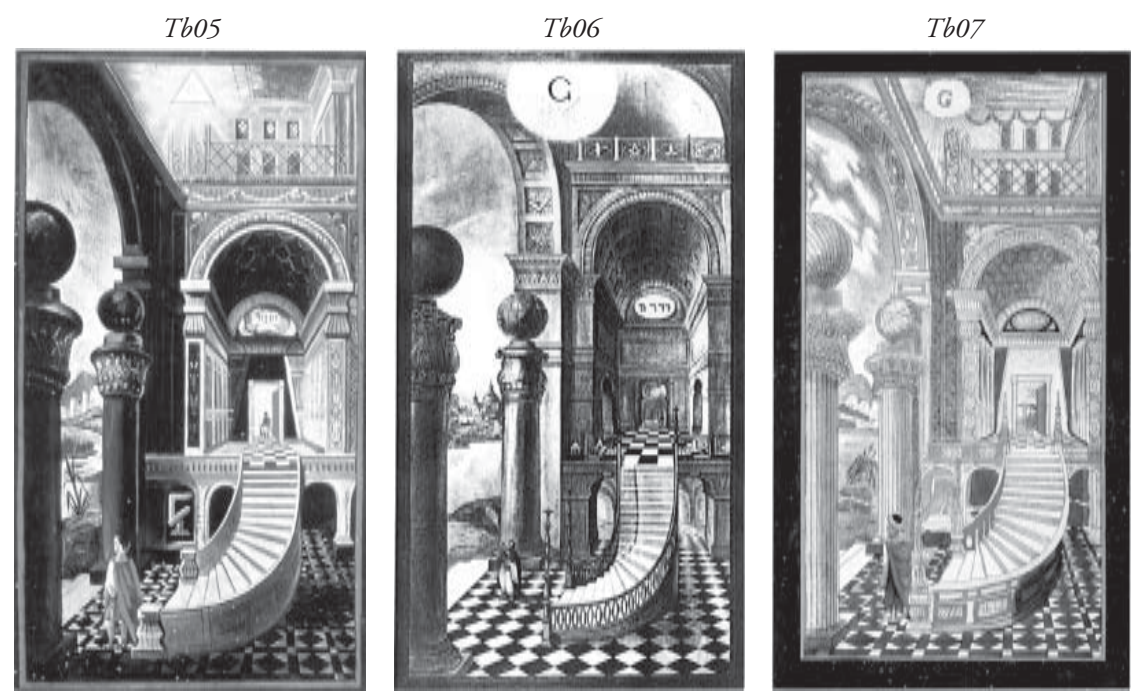

périeure occupe une surface importante. Ces portions d'un même Tableau font partie de l'objet total pour au moins deux raisons: elles sont coprésentes dans la surface globale, délimitée par un seul et même cadre; elles sont séparées par un trait de même couleur ou/ et de même largeur, c'est-à-dire de même densité et de même importance. Ce trait est lui-même entouré par le cadre «réel», ou définitif. Lorsqu'il est unique ou qu'il se présente comme un sur-cadre, il indique sur des supports divers, planches, pièces de linge, toiles (à peindre). Mais tous ont été exécutés selon la même forme. La matière du support importe peu, en vérité, puisque la reproduction d'un Tableau sur papier n'enlève rien au mode de lecture ou d'occupation de la surface. Il semble donc y avoir une homologie forte entre le Tableau et son support. L'orientation du Tableau aura des conséquences sur la signification qu'on lui donnera.

L'élément principal qui le caractérise est un/son cadre. C'est à l'intérieur du cadre que l'objet acquiert ses caractéristiques. Si un Tableau sans cadre apparent (cadre au degré zéro) pourrait rester un Tableau en raison des éléments qui le composent, un cadre seul, autour d'une surface vide, ne suffit pas à créer un Tableau. Le cadre est la trace matérialisée d'un bord, et la matérialisation de la fin d'une surface désignée comme signifiante. Il renvoie à la fin de l'espace particulier qu'il délimite en le créant. Ici, le cadre peut n'être qu'un trait, plus ou moins épais, ou une combinaison de traits. Il peut aussi servir d'ornement (Tb01 orné de frise, ou Tb02 dont le trait noir aux coins ornés de pompons l'assimile à un cordon). Beaucoup de cadres sont des combinaisons de traits. Si le Tb03 est entouré d'un trait, le Tb07 a deux cadres imbriqués. Celui du Tb09 est fait de six traits. Le principe est retenu avec le $T b 10$.

Le cadre est-il présent seulement par une habitude culturelle d'encadrement (artistique) ou possède-t-il d'autres fonctions? Il n'est pas, ici, la représentation du cadre qui peut entourer un tableau, comme partie rapportée, cadre fait d'un autre matériau, une baguette de bois travaillée. Le cadre du Tableau de Loge fait le plus souvent, semble-t-il, partie intégrante de l'image. Il est une partie du Tableau.

Dans plusieurs d'entre eux, on trouve un découpage de la surface en deux parties. Pour le Tb03, une partie inférieure, plus petite, est inscrite dans toute la largeur. Pour d'autres («Harris $2^{\mathrm{e}}$ période», Tb10), la partie su-

l'intention préalable de l'auteur ou du commanditaire. Sa forme épouse le bord, réel ou supposé, d'une étendue de matière dotée, dans notre culture, de propriétés particulières. Sa rectangularité, composée de lignes parallèles deux à deux, d'angles identiques à force d'être droits, sont, en Occident, des marques de régularité, de stabilité, de rationalité. Le cadre délimite et crée donc un espace rationnel. C'est dans cet espace que les éléments seront structurés et disposés. Ils font partie d'une sorte de «fenêtre» qu'on a délimitée pour nous les présenter, en excluant toute échappée hors-cadre. Ce qui est à voir, et rien d'autre, est enfermé dans cet espace et cette forme.

Celle-ci dérive, en fait, de la «forme de la Loge», espace le plus souvent rectangulaire et orienté. On découvre cette orientation, de manière apparente, sur un Tableau (Tb01) où on a indiqué les initiales des points cardinaux. L'orient en haut (E pour East) détermine les autres coordonnées. Tous les Tableaux présentent une disposition identique car, même si les initiales manquent, il semble assuré qu'elles sont sous-entendues. Espace "à part" par son cadre et sa forme, le Tableau l'est donc encore par son rapport à la Loge dont il épouse, reprend ou exprime, certaines contraintes spatiales. L'espace enclos dit déjà une structure.

La structure du Tableau est évidemment géométrique. L'évidence semble aussi naturelle que peu sensible à l'analyse. Considérons cependant les axes principaux de la figure, les médianes, les diagonales, et leur point de croisement, le centre. L'utilisation de la géométrie n'est, en réalité, jamais seulement une habitude. Elle suppose une intention: décrire le monde, de manière ordonnée et rationnelle. La forme rectangulaire répondait à cette intention, les axes la confortent. Sa fonction de structuration amène, en outre, à réfléchir sur la présence d'une grille de traçage du Tableau. La disposition des éléments implique donc une intention supplémentaire dans la manière d'orienter le regard du lecteur, de le faire se heurter à une forme 
régulière, à un cadre, à des angles droits. L'enfermement d'éléments, reprenant des objets du monde, réels ou imaginés, dans une telle grille, révèle donc cette intention. C'est la mise en place d'un système et la création d'un «ensemble», l'unité d'un énoncé.

\section{Description et composition}

Dans notre culture, une image est lue, en général,
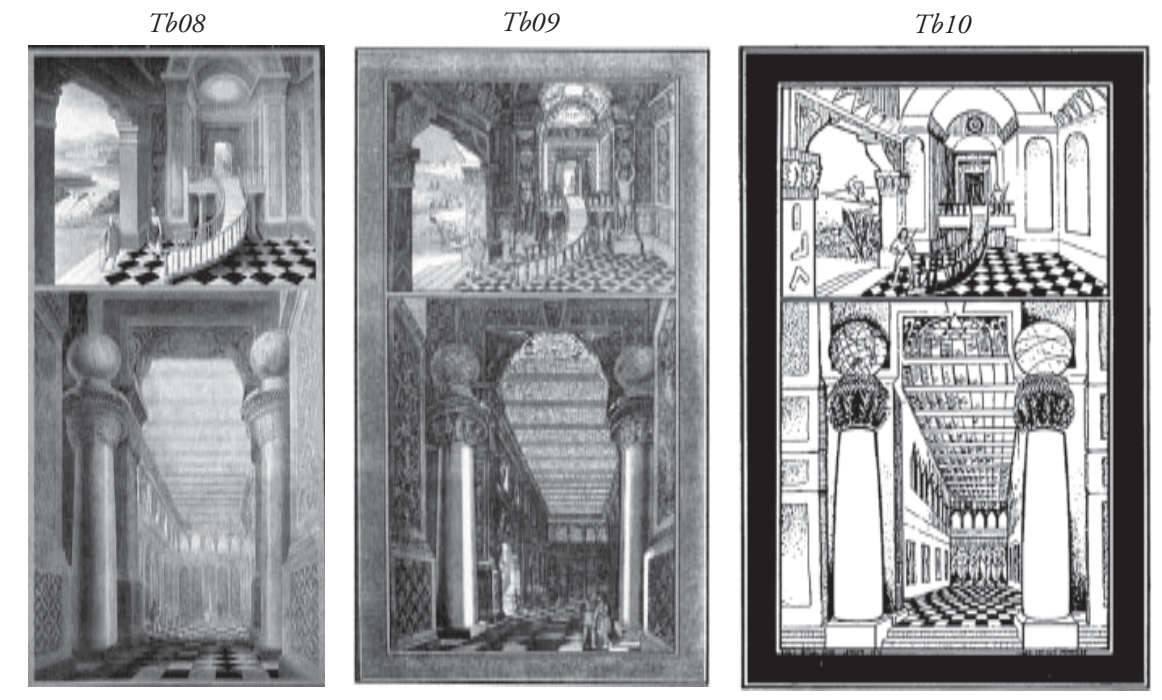
de gauche à droite, comme un discours écrit. Le lecteur aura donc tendance à considérer les éléments d'une unité de sens selon une succession répondant à cette démarche et à essayer d'y percevoir la structure constituée. La tendance est loin d'être naturelle, mais l'habitude nous semble une seconde nature. Dans cette logique, la question se pose, immédiatement, de savoir si l'auteur a disposé les éléments selon ce schéma. Pour un auteur de la même aire culturelle, la réponse sera probablement positive. Nous admettrons donc ce processus, partagé par l'auteur et son lecteur, ici le peintre et les Maçons. Le sens de l'image naîtra de la disposition que nous tenterons de décrire pour en trouver les articulations. Quels éléments se trouvent donc dans les images? Quels éléments communs? Comment sont-ils disposés? De quoi sont faites leurs différences?

Les deux premiers Tableaux sont assez simples. Ils sont dessinés de manière rudimentaire, à la limite de la schématisation. Le Tb03 comporte un début de mise en scène, en particulier dans sa deuxième partie. De plus, l'usage de la couleur, la technique, le souci du détail, le font entrer dans une catégorie proche du tableau artistique. Les autres Tableaux, même le Tb04 assez dépouillé, présentent des aspects artistiques indéniables: souci de composition, technique sûre et assez classique (sauf pour le Tb07, qui est la reproduction malhabile d'un Tableau de Harris). En première vision globale, les Tableaux représentent une scène où des personnages sont dans un bâtiment, ou à proximité de celui-ci. Cela rend déjà compte de certaines constantes et reproduit des notions appartenant à l'expérience commune (l'encyclopédie).

L'élément qui attire d'abord l'attention est un escalier occupant une partie décisive de l'image. On constate ensuite la présence, imposante, de deux colonnes, supportant des globes, puis celle d'un bâtiment, de plusieurs personnages, d'un végétal et d'une chute d'eau. L'escalier est placé sur un sol (ou en relation avec celui-ci) dont la

surface est particulière. L'édifice peut être vu de l'extérieur mais, le plus souvent, on en voit l'intérieur. Les deux colonnes sont en rapport de contiguïté directe avec le bâtiment et, de manière aussi systématique, on voit des personnages proches de l'escalier. On trouve enfin un élément n'appartenant pas à l'architecture mais qui est connu comme symbole du sacré. Une irradiation est, en effet, présente sous différentes formes, et parfois même répétée dans un même Tableau.

\subsection{Le groupe $S$}

Un groupe d'éléments, que nous appellerons "groupe S», semble bénéficier d'un statut particulier. Il est composé d'une plante et d'une chute d'eau. Dans les premiers documents, ces éléments sont nettement réunis et sont à l'extérieur de l'ensemble de tous les autres. Ils peuvent être en haut à droite (Tb01), ou en haut à gauche (Tb02). Même placé à l'intérieur du cadre, il est net que ce groupe ne fait pas partie de la «scène» principale, tout en ayant un rapport avec elle. La place qu'il occupe dans les autres images semble le confirmer. Dans quatre Tableaux, il est dans un espace particulier, une sorte de vignette (relativement) indépendante. Mais, plus intéressant, dans les Tableaux à image unique, il est situé en dehors de l'espace principal qu'est l'édifice. Tout concourt à souligner son rapport avec le reste de l'image.

\subsection{L'échappée sur le monde extérieur}

Le plan général du Tableau, centré sur l'intérieur du bâtiment, laisse une échappée, de biais, vers le monde extérieur dans lequel on fait exister l'édifice. Cet espace est situé à gauche ou, pour un lecteur, au début de son balayage visuel, là où il intègre les premiers éléments de sens. Cette disposition est dans un rapport synonymique avec les vignettes et même avec les éléments groupés des Tb01-02. L'échappée est presque hors cadre. Si quatre Tableaux 
présentent des vignettes horizontales, les autres proposent des bandes verticales, assez semblables, finalement, dans leurs rapports avec l'image centrale.

Ce qu'on a voulu représenter dans cette échappée va du plus schématique au plus organisé. Pour le Tb03, c'est un véritable paysage de style romantique et aucun autre Tableau n'atteint ce développement. Certains éléments sont pourtant communs à tous. D'abord, une représentation de la nature; souvent, celle de la présence de l'homme et de son activité; plus rarement, des êtres vivants. Cela permet d'ancrer cette partie de l'image dans un monde «réel», ou d'ancrer toute l'image dans une «réalité». Deux éléments y sont toujours présents: ceux qui constituent le groupe $S$.

La vignette, ou l'échappée, comme ce groupe, sont une représentation d'éléments de la nature. De plus, leur emplacement les met à distance du cour du Tableau, autant qu'en rapport avec lui. Cela crée, ainsi, une relation d'opposition «extérieur» versus «intérieur», qui est aussi une opposition «nature» versus "culture», terme représenté par l'édifice. Les Tableaux soulignent cela par un élément de transition entre l'extérieur et l'intérieur du bâtiment: des marches d'escalier, appartenant à l'édifice mais placées audehors.

\subsection{Le bâtiment}

Tous les éléments, scènes ou personnages, sont liés en effet à une structure architectonique en plusieurs parties, dont l'escalier et deux colonnes toujours nettement visibles. L'édifice est plus ou moins imposant et décrit avec plus ou moins de détails. Dans deux Tableaux, il nous est présenté de manière abrégée ou schématique: le Tb02 propose seulement «la porte» du temple, pour dire «le temple dans sa totalité»; le Tb01 présente un "petit temple». Mais le temple et les colonnes qui sont à gauche de l'image marquent les deux points extrêmes d'un trajet: un début avec celles-ci et son terme avec celui-là.

L'idée que l'édifice est un temple se renforce avec le Tb03: les doubles colonnes supportent un fronton triangulaire, rappel des temples classiques. La représentation, aussi éloignée que possible d'une restitution archéologique, reprend assez de principes d'architecture pour évoquer simplement «un temple». De plus, les colonnes qui encadrent l'image sont très massives par rapport aux autres éléments: elles sont au premier plan, devant le temple. Il faut sans doute y voir une relation directe entre temple et colonnes, qui est confirmée par ailleurs: d'une part, l'édifice occupe la totalité de la surface, en une ou deux images; d'autre part, les colonnes sont placées à l'entrée de l'édifice ou devant lui. À part les trois premiers, les Tableaux montrent l'intérieur d'un édifice que ses détails, éléments et dispositions permettent donc d'appeler temple. Le personnage placé dans l'entrée constitue la "norme humaine», l'échelle; la norme qui permet ainsi de montrer que l'entrée comme l'intérieur de la construction sont hors-norme, monumentales et même colossales. Ces dimensions renforcent l'idée que l'édifice n'a pas pour but d'abriter des activités ordinaires. Un temple...

Un élément frappe par sa position centrale et par ses caractéristiques physiques (forme et position). Il s'agit d'un escalier qui est toujours placé entre une ouverture, située au niveau zéro (N0), et un élément placé à un niveau supérieur (N1). L'intérêt réside dans le fait que l'escalier fait face à une entrée béante donnant sur l'extérieur, et qu'il occupe en quasi-totalité la largeur de l'entrée, en arrière de celle-ci, à l'intérieur. Tout en étant sans doute un élément extérieur, il est tout aussi bien une partie de l'édifice. Sa composition est diverse, mais sa forme est toujours particulière. Il décrit un arc de cercle entre sa première et sa dernière marche. Il commence en général à gauche de l'image et suit, ainsi, le sens de la lecture, ce qui le rend plus apte à accrocher le regard et plus facilement décodable. En tenant compte de l'orientation des Tableaux, on dira qu'il part du nord et qu'il finit vers l'est. Dans tous les cas, il se termine au centre de l'image et même, assez souvent, directement sur la médiane, sinon, au centre de l'espace du bâtiment.

Dans tous les Tableaux, il existe un élément de liaison entre l'escalier et l'entrée. Cette liaison se manifeste par l'utilisation d'un sol particulier, fait de damiers le plus souvent, sur lequel repose l'escalier et qui sert, par des effets de perspective, à indiquer une tension vers l'avant. On guide le regard vers l'endroit où s'arrête le damier. L'escalier, posé sur un tel pavage, s'élève vers un plan second, toujours situé à l'intérieur de l'édifice. Il peut être directement placé au bord d'une entrée (Tb02) ou séparé de celle-ci par un plan incliné intermédiaire fait d'un pavage en damiers (Tb01). C'est, sur le plan horizontal, un rectangle qui s'allonge devant le lecteur, jusqu'à l'autre entrée située au fond du vestibule. La perspective permet de situer l'entrée au bout d'un espace qui va se resserrant, 
dans une forme d'entonnoir. Le vestibule mène vers une entrée en arrière-plan. L'effet d'entonnoir est soutenu par les lignes de fuite. Le resserrement existe dans le Tb01, dans le $T b 02$ avec les carreaux du sol (N0), et dans le TbO3 avec les murs du temple encadrant un petit vestibule. Le sol des couloirs des Tableaux Harris est à peu près au niveau du plan de vision du lecteur, qui occupe ainsi un point de vue plutôt élevé par rapport à l'entrée principale. Le lecteur est alors placé au centre du volume créé par la perspective, face à la porte, et souvent en vis-à-vis par rapport au personnage qu'on devine au fond du couloir. L'attention a été guidée dans une ascension de l'escalier jusqu'au fond du corridor.

À côté d'un escalier généralement central, l'importance des colonnes ne peut être niée. Dans le Tb01, c'est l'élément perçu en premier. Dans le Tb02, elles dominent l'escalier. Dans tous les autres, elles sont un élément déterminant de la composition comme du temple. Elles peuvent être face au lecteur (Tb01-03 et Tb08-10). Elles peuvent encadrer la composition (Tb03), le passage (Tb01) ou l'entrée (Tb08-10). Elles peuvent être de biais, sur une ligne de fuite (Tb01). Dans les Tb04-10, elles encadrent l'entrée et font partie de l'édifice. Sauf dans les Tb08-09, où elles sont entièrement dans le bâtiment, elles sont à la fois à l'intérieur et à l'extérieur. Mais il est net qu'elles ne jouent aucun rôle réellement architectonique, car ce sont des colonnes qui ne soutiennent rien. L'édifice pourrait conserver sa stabilité sans elles. Elles sont pourtant présentes.

On remarque leur parfaite similitude, sauf dans le TbO1 où elles sont affectées de styles différents. Elles ont donc en général même taille, même forme, même style, mêmes chapiteaux, mêmes bases, mêmes ornements. Un seul élément les différencie: les globes qui les surmontent. Ordinairement de même volume, ils sont pourtant différents. L'un est une sphère céleste, l'autre un globe terrestre. Les globes du Tb03 sont, de plus, couverts d'une sorte de filet. Les colonnes et leurs globes sont parmi les premiers éléments rencontrés par le regard. C'est, de plus, entre les colonnes qu'on aperçoit l'échappée vers l'extérieur et qu'a été placé l'escalier. Elles opposent leur verticalité au déplacement horizontal qui va de l'extérieur vers l'intérieur. Elles sont l'élément séparateur qui délimite, mieux que l'entrée, extérieur et intérieur. La prise de conscience de leur présence "transporte» à l'intérieur de l'édifice, où se trouve la quasi-totalité des objets.

\subsection{Les personnages}

Au long du parcours, le lecteur est confronté à trois personnages, remarquables, qui sont positionnés à l'intérieur du bâtiment. Deux d'entre eux semblent entretenir des liens particuliers. Ils sont en effet dans une configuration spécifique, et nous dirons qu'ils "vont ensemble». Dans deux Tableaux (Tb08-09), on aperçoit un troisième personnage; dans certains autres, jusqu'à quatre personnages. Le premier personnage principal (P1) est toujours placé au bas de l'escalier (devant ou sur la première marche) et il fait toujours face à l'entrée. Il semble donc attaché à l'entrée et/ou au début de l'escalier. Le deuxième (P2) est en une sorte de symétrique. Il est toujours placé après l'escalier (directement sur la dernière marche, ou plus en arrière). Mis à part le Tb10, $\mathrm{P} 2$ semble lié à la porte figurée au centre de N1. De nombreux points communs existent entre P1 et P2. Ils portent des ustensiles qui semblent identiques, leurs vêtements sont assez semblables. Ils peuvent, tous les deux, être revêtus d'un tablier maçonnique (Tb03-02). Leur placement permet de les différencier, mais aussi le bijou qu'ils portent au cou (Tb03). En résumé, si P1 semble garder (et contrôler) le début de l'escalier (ou le départ d'un parcours ascensionnel), P2 semble garder (et contrôler) la fin du parcours (et l'entrée au fond du vestibule). Enfin, leur attitude est enfin à la fois commune et particulière. Ils semblent toujours immobiles et laissent penser qu'ils attendent (quelque chose ou quelqu'un).

Deux Tableaux (T608-09) proposent un troisième personnage (P3), à gauche de l'entrée, dans une attitude qui indique, au contraire, qu'il est en mouvement, et même qu'il va de l'extérieur vers l'intérieur. Il fait face à P1 comme à l'escalier. On remarque aussi que P3 est placé sur la médiane de l'entrée comme de l'escalier. C'est, ici, le seul en mouvement. Il regarde, de plus, vers l'avant (vers la droite dans le sens de lecture) ou vers P1 qui semble lui tendre la main. Dans ces deux Tableaux, on distingue quelques personnages supplémentaires, mais leur éloignement de l'espace central fait davantage penser à des indications périphériques.

\subsection{Les symboles divins}

À côté des proportions du bâtiment, quelques détails appuient l'idée qu'il puisse s'agir d'un temple: d'abord des putti, angelots présents dans quelques Tableaux et, surtout, des symboles du divin en nombre important. Ils se mani- 
festent le plus souvent par une irradiation, qu'on appelle techniquement "gloire» ou «nimbe». Elle existe sous différentes formes et peut inclure ou s'associer à d'autres éléments: d'abord, une figure géométrique, cercle (Tb06, 07, $10)$, triangle $(T b 01,05)$, ou hexagramme (Tb04); ensuite, l'œil divin ${ }^{2}$; un ou plusieurs caractères hébraïques. Au nombre de quatre, ils forment le tétragramme $(Y H W H)$, du nom de Dieu. Un seul caractère représente la première lettre du tétragramme; une lettre latine, un $G$; pour terminer, une colombe tête en bas qui, dans la culture chrétienne, est un symbole classique de l'Esprit saint ${ }^{3}$. Il y a souvent coprésence de deux symboles, plus rarement de trois. Le Tb04 présente la plus grande redondance: on trouve à la fois l'hexagramme, les lettres hébraïques, la colombe et les angelots.

Tous ces symboles, comme leur positionnement, sont les indices d'une relation de l'édifice avec le religieux ou le spirituel. En aucun cas, en effet, des obligations de composition artistiques ou architecturales n'obligent à les représenter. Ils sont, de plus, tous placés en hauteur et, d'une manière ou d'une autre, au centre de l'image. C'est même le premier élément qu'on découvre sur le Tb01. Dans le Tb03, cette place est occupée par l'œil divin avec, sur la médiane, un caractère hébraïque nimbé. Dans les Tb0407, un élément est sur la médiane du Tableau, un (ou deux) autre(s) sur celle du vestibule. Dans les Tb08-10, ils sont sur la médiane du vestibule ou le linteau de la porte. On constate qu'ils dominent les compositions dans une position hiérarchique forte qui influe sur ce qui se trouve au-dessous. Ce ne sont pas des éléments de décoration au sens ordinaire: leur placement, leur statut de symboles du divin introduisent un élément, qui va coordonner l'ensemble et suggérer la présence divine dans le bâtiment et même dans la composition entière. Les mêmes symboles, en relation avec des portes, suggéreront cette présence dans la pièce située derrière l'entrée (Tb03). Si la présence divine est générale, elle est renforcée dans un lieu (quand l'entrée est franchie) qui devient un espace particulier, séparé. C'est une manière de représenter le «lieu de la shekel ${ }^{4}$ de la tradition hébraïque.

Une dernière remarque s'impose. Les éléments nimbés sont mis en rapport de synonymie, mais les figures géométriques dénotent aussi l'usage d'une technique particulière, l'art de la géométrie. On peut l'oublier avec l'hexagramme, qui n'est souvent perçu que comme symbole du divin, avec le triangle, symbole habituel de la Trinité, ou avec le cercle, symbole le plus simple de totalité, et particulièrement du monde spirituel. Mais les combinaisons seraient différentes: le triangle pourrait contenir l'œil et non la lettre $G$, l'hexagramme serait dessiné seul. Il y a création d'un axe paradigmatique sur lequel sont placés les divers symboles et aussi la science de la géométrie.

\subsection{Les symboles de métier}

Nous distinguerons un dernier groupe d'éléments composé des outils du métier. Si la géométrie est présente dans tous les Tableaux, elle n'est pas nécessairement exprimée de manière audible. D’autres moyens sont employés qui sont aussi efficaces. C'est ainsi que, dans la majorité des compositions, on constate la présence des outils, symboles de métier. Ils peuvent orner le fronton de l'édifice, ou être posés sur le sol (Tb03). Dans ce cas, leurs dimensions sont loin de celles d'outils ordinaires. Ils peuvent être dessinés dans des cartouches (T604,05 et 07), ou orner une balustrade ou l'entrée principale (Tb08). De plus, deux outils sont directement liés à $\mathrm{P} 1$ et $\mathrm{P} 2$. Dans le Tb03, ils sont portés à la manière de bijoux, suspendus à un collier. P2 peut encore tenir un niveau (Tb05, 08 et 09). On supposera que $\mathrm{P} 1$ pourrait tenir un fil à plomb.

Tout cela permet de rappeler plusieurs notions: le bâtiment est le lieu de la présence divine; il est le résultat de l'activité humaine, celle-ci est liée à un métier, et en même temps elle l'abrite; enfin, les outils précisent sa nature, la construction. L'intégration, l'imbrication de tous les éléments suggère la coexistence, le rapport, la corrélation et la dépendance des articulations: édifice, divin, métier.

\section{Le point de vue et les axes}

La composition met le lecteur face à une scène. Dans les trois premiers Tableaux, il est à l'extérieur d'une construction, présentée de face. Pour tous les autres, le point de vue imposé le place quelque part à l'intérieur, face au plan principal et perpendiculairement à l'entrée à gauche. Il est, de plus, légèrement en hauteur, à peu près au niveau de la porte visible au fond du vestibule. Ce point géométrique est au croisement de deux axes. Nous remarquons ainsi une structure générale conçue selon nos schémas de lecture imposant un premier balayage de gauche à droite. On est incité à pénétrer, mentalement, l'image par l'entrée grande ouverte, à gauche, en ayant d'abord lu les globes 
énormes placés sur des colonnes monumentales. On rencontre ensuite l'escalier et, enfin, on le remonte vers le centre de l'image. Cela nous conduit à évoquer les axes qui charpentent les Tableaux et qui les structurent selon des axes orthogonaux.

Le regard du lecteur était d'abord attiré par l'irradiation présente au centre du plafond, exactement sur la médiane verticale. D'autres verticales importantes existent, en particulier dans les Tb04-10: l'une d'elles passe au milieu des colonnes, une autre au milieu de la voûte de l'étage. Sur la première, sont placés des éléments de la scène extérieure; sur l'autre, de haut en bas, le milieu du plafond, une fenêtre, le centre de la voûte, le tétragramme, la porte, un personnage (éventuel), le milieu de l'escalier et le point où celui-ci infléchit sa direction. Toutes ces lignes influeront sur l'impression de verticalité des Tableaux et valorisent un axe général, avec l'idée d'ascension que suggère la majeure partie de l'escalier. Cette dernière notion permet de souligner un point qui n'avait pu être abordé plus tôt: si l'axe vertical permet de placer les objets énumérés "de haut en bas", dans une lecture habituelle, il est clair, après ce bref parcours, qu'il faudra remonter la verticale, de bas en haut cette fois, pour trouver le codage interne des Tableaux.

À côté des axes verticaux, d'autres éléments proposent des parcours horizontaux très nets: l'entrée et l'escalier organisent une horizontale gauche-droite. S'y trouvent le milieu d'une entrée, P1, le milieu de l'escalier. La structure $\mathrm{du}$ sol avec ses carreaux, quelles qu'en soient les formes, matérialise l'axe et le renforce. Un deuxième axe existe, bien qu'un peu moins sensible: le haut de l'escalier avec les balustrades marque une horizontale à un niveau, parallèle au sol. Un troisième axe est marqué par le haut des murs soutenant la voûte du vestibule. La balustrade qui surmonte cette voûte en marque une quatrième (Tb0407) et on pourrait en trouver une cinquième avec les caissons du plafond. On obtient, par le croisement des axes, une sorte de quadrillage qui fait apparaitre des points de croisement, des noeuds, où sont placés certains éléments. La première horizontale forme avec la verticale un parcours en équerre: on passe du sol (plan horizontal), à une montée (plan vertical), par l'escalier qui décrit un quart de cercle. Celui-ci participe alors des deux axes. Il est même l'élément de transition entre les deux.

L'usage de la perspective permet de déceler encore un axe horizontal n'appartenant plus à la logique tabulaire de l'image, puisqu'il n'est pas parallèle au cadre. Le haut de l'escalier et la porte sont placés sur une horizontale «mentale», en ce qu'elle pénètre l'image en s'éloignant du lecteur. La structure du sol carrelé et les lignes de fuite renforcent cet axe et accentuent l'effet d'éloignement. Remarquons qu'il est confondu avec la verticale qui va du bord inférieur du Tableau au centre de la porte, et même jusqu'au centre de la voûte la surmontant, puisqu'elle est sur une ligne de fuite. La perspective a été utilisée, de manière globale, pour créer l'impression de profondeur et de volume, pour créer des différences dans les parties de l'image, et aussi pour donner l'impression de vérité. Le point de fuite est au deux tiers de l'image en largeur et au milieu en hauteur. Tout est structuré pour conduire le regard vers un point, au centre de l'image, situé en haut de la porte.

\subsection{Le jeu des oppositions}

Malgré l'importance de l'axe de lecture, il semble donc nécessaire de chercher le sens des compositions à partir du bas de l'image, sur l'axe horizontal d'abord et en remontant. Il avait cependant semblé évident que l'irradiation dominait les Tableaux (pour le Tb02, c'est la clé de voûte qui en tient lieu). C'est donc elle qui, malgré tout, semble donner le sens général. Les différents éléments peuvent ainsi permettre d'identifier une série d'oppositions comme, d'abord, début/fin, périphérie/centre, dehors/dedans, nature/culture, bas/haut, horizontal/vertical.

Il y a début et fin d'un parcours (du monde hors temple à l'espace hors vue situé derrière la porte du vestibule). L'itinéraire va du monde extérieur (périphérique) au centre, par la porte, au centre du Tableau. On va du dehors au dedans, du monde naturel au monde de la construction (le temple). On entre alors dans une opposition plus générale nature/culture. On va encore du bas, le sol, vers le haut, l'étage (et sans doute plus), en remontant la verticale jusqu'à son terme, on va d'un monde horizontal (passage de l'extérieur à l'intérieur) à un monde vertical (passage du bas vers le haut) par l'escalier, placé au confluent des deux dimensions. Ces oppositions en amènent d'autres comme bruit/silence, mouvement/immobilité, 
où le bruit est la nature figurée par le fleuve et l'eau en cascade (chute d'eau), le vent dans les feuilles, et où le silence règne dans le temple (œuvre d'homme). Comme pour renforcer l'opposition bruit/silence, on ne décèle pas de réel mouvement (personnages en attente, ou arrêtés), ce qui s'oppose à la dynamique de l'eau. Dans l'édifice lui-même, on remarque une opposition anguleux/courbe correspondant à l'opposition bas/haut. L'irradiation, symbole du monde céleste, permet de risquer l'opposition terrestre/céleste, où celui-là peut contenir bas, horizontal et anguleux.

\section{Le texte rituel}

L'appartenance des images au deuxième grade en FrancMaçonnerie rend probable une référence au texte du rituel et au discours qu'il libère. Il est probable aussi que certains éléments ne s'expliquent que de cette manière, ou qu'ils s'expliquent mieux ainsi. La relation entre Tableau et rituel est déjà exprimée par les outils, symboles du métier. Il semble donc qu'un détour par le texte rituel ne soit pas inutile.

\subsection{L'édifice}

L'édifice à l'intérieur duquel on a placé le lecteur est le Temple de Salomon. Ce n'est pas exprimée directement par les Tableaux, mais par les rituels. L'accoutrement vaguement oriental de nombreux personnages sert sans doute à suggérer une contrée lointaine et exotique, un Orient mythique. Nous savons que c'est autour du Temple que les rituels ont élaboré leurs légendes fondatrices. Le Temple, sa construction, son commanditaire sont bien sûr des points relatés par la Bible ${ }^{5}$, Livre des Rois (1 R 7, 15-22, 40-42 et 46) et Livre des Chroniques (2 Chr 3, 15-17 et $2 \mathrm{Chr} 4$, 11-13, 16-17), en particulier.

Les rituels y insistent longuement et font, de plus, état d'un luxe de détails qui ne sont pas toujours dans la Bible. Si un élément (le Temple) est emprunté au texte biblique, il faut nuancer l'affirmation. L'emprunt n'est pas toujours direct. Il a surtout été fait par l'intermédiaire de commentaires. La Bible ne propose pas, en effet, les interprétations des rituels et en particulier celles qui sont illustrées par les Tableaux. Il faut de plus replacer le texte biblique dans son contexte. Sans point de référence scientifiquement assuré, le Temple et ses diverses parties ont été imaginés en se fondant sur une traduction répandue mais déjà ancienne, la King James Version 6 (KJV, 1611). Le rituel indique d'abord que "When the temple at Jerusalem was completed by King Solomon... ». La KJV relate l'intention de Salomon ainsi: «I purpose to build an house unto the name of the LORD my God...» ${ }^{7}$ puis, à l'achèvement du Temple, elle indique: «So ended all the work that King Solomon made for the house of the LORD...» 8 .

L'édifice est donc bien le Temple de Salomon mais il est imaginé et conçu par les lecteurs et les commentateurs de la Bible et, en conséquence, par les Maçons, qui peuvent appartenir à l'une et l'autre catégories. La configuration de l'édifice va s'en ressentir et les usages qu'on attribuera à telle ou telle partie le seront selon la perception du texte, d'abord, selon les images qui en seront tirées, ensuite. Rien dans ce qui est proposé ne peut faire référence à des données archéologiquement certaines. La Bible ne mentionne pas ce que proposent certains Tableaux: pas d'arc entre les colonnes, pas d'escalier incurvé, pas d'escalier à l'intérieur du Temple, pas de pavage en damiers, pas de personnages contrôlant les entrées. Ce temple est à peu près totalement imaginaire et n'est que l'image, canonique, d'un temple pour figurer le Temple. Il semble pourtant assuré que les Maçons et les illustrateurs estimaient leur représentation juste.

\subsection{Les colonnes}

Les colonnes seront donc celles du Temple de Salomon. La Bible indique qu'elles étaient devant le bâtiment. Pour les Maçons, ce seront celles du Temple où qu'elles soient placées. Les premiers Tableaux présentent ainsi des colonnes vaguement classiques, placées devant l'édifice. Les autres les affectent d'un style rappelant l'égyptien, ou l'oriental, plutôt engagées dans le bâtiment. C'est ainsi que les lecteurs ont perçu la phrase: "The pillars in the porch of the temple». Elles sont ainsi souvent dans l'entrée. Des traductions plus récentes diront: «On dressa les colonnes devant le Sanctuaire " ${ }^{9}$, ou «Hiram érigea les deux colonnes devant le portique du Temple» 10 .

Rien non plus dans l'agencement biblique ne semble suggérer les globes des Tableaux. Il faut reconnaître que la King James Version donnait:

12 To wit, the two pillars, and the pommels, and the chapiters which were on the top of the two pillars, and the two wreaths to cover the two pommels of the chapiters which... ${ }^{11}$

Si le verset permet les globes identiques des deux premiers Tableaux, il n'autorise pas ceux des autres qui sont des globes célestes et terrestres. Le rituel a donc rajouté: 
Those Pillars were further adorned with two spherical balls on which were delineated maps of the celestial and terrestrial globes... ${ }^{12}$

Rien n'est ici du domaine biblique et il est permis d'y voir un ajout tardif qui doit tout à la Maçonnerie et à la culture scientifique du XIX ${ }^{\mathrm{e}}$ siècle naissant.

\subsection{L'escalier et le plan supérieur}

Comment l'escalier s'insère-t-il dans cette explication? Le texte rituel expose que:

At the building of King Solomon's Temple [...] the fellowcrafts were paid their wages in specie, which they went to receive in the middle chamber of the Temple. [...] After our ancient Brethren had entered the porch, they arrived at the foot of the winding staircase which led to the middle chamber. ${ }^{13}$

Les Tableaux tentent d'illustrer ce qu'affirme le rituel avec le plus d'exactitude possible, car leurs auteurs ont essayé de rendre en image ce que les mots du texte leur suggéraient. C'est la raison de l'escalier "tournant", mais pas du quart de cercle visible dans les Tableaux. Quant à la Bible, elle évoque bien un escalier, mais celui-ci n'a jamais de forme précise:

The door for the middle chamber was in the right side of the house: and they went up with winding stairs into the middle chamber, and out of the middle into the third. ${ }^{14}$

Il pourrait tout aussi bien s'agir d'un escalier en colimaçon comme de n'importe quel escalier, qui par nature "tourne», à la différence d'une échelle.

Un texte plus récent nous présente en effet les choses ainsi:

On accédait au rez-de-chaussée par une porte sur le côté sud du temple; de là on montait à l'etage intermédiaire par des escaliers tournants, puis de même à l'étage supérieur ${ }^{15}$,

ce que la Bible de Jérusalem traduit par: «L'entrée de l'étage inférieur était à l'angle droit du Temple, et par des trappes on montait à l'étage intermédiaire, et de l'intermédiaire au troisième». La Traduction oecuménique de la Bible choisit de conserver cette notion de "trappes»:

L'entrée de l'annexe inférieure était vers le côté droit de la Maison. Par des trappes, on pouvait accéder à l'annexe $d u$ milieu et, de celle du milieu, à la troisième.
On précise en note qu'étage de milieu, même s'il est littéral, est "incompréhensible», et qu'on retient "inférieur, d'après le grec".

Pour les créateurs des Tableaux, lisant une traduction particulière de la Bible, le Temple était réellement doté d'un escalier menant à une salle dite "chambre du milieu ", et, grâce au rituel, ils ont affecté à ces éléments une utilisation symbolique précise, en rapport direct avec leurs préoccupations. La classe intermédiaire des ouvriers (ou "du milieu »?) avait droit d'accès à la salle du milieu, située spatialement entre une inférieure et une supérieure, comme les Compagnons le sont entre Apprentis et Maîtres. Heureuse coïncidence, pleine de ressources. Les rituels se sont aussi arrêtés sur la notion de milieu (middle chamber), qui reprend la fonction symbolique du centre. On comprend alors pourquoi la porte représentée au fond du vestibule est placée au centre du Tableau. La porte comme la "chambre» placée derrière sont visiblement affectées de toutes ces notions de centre, avec l'ensemble de l'aspect sacré que le symbolisme permet de suggérer.

Il reste probable, quant aux Tableaux, que la forme particulière de l'escalier ait été suggérée par le poids symbolique de l'outil «équerre» qui, en Maçonnerie, fait partie des «trois grandes Lumières». Il faut malgré tout reconnaître que cette forme est dotée, sur le plan graphique, d'avantages qui ont permis aux auteurs de faire partir l'escalier d'un côté de l'image (le plus souvent à gauche), pour le faire s'élever face au lecteur et pour évoquer ainsi une ascension. C'est, en termes d'effort, la forme de transition entre deux niveaux la plus économique.

\subsection{Les personnages}

Comme il en est des éléments précédents, seule la lecture du texte rituel permet de comprendre réellement la présence de $\mathrm{P} 1$ et $\mathrm{P} 2$, «en attente» à chaque extrémité de l'escalier. La description permettait d'en avoir quelques indices mais le fondement biblique de leur présence pouvait nous échapper. Citons le rituel de manière plus complète:

After our ancient Brethren had entered the porch, they arrived at the foot of the winding staircase which led to the middle chamber. Their ascent was opposed by the Junior Warden, who demanded of them the Pass Grip and Pass Word leading from the First to the Second Degree. [...]

After our ancient Brethren had given those convincing proofs to the Junior Warden, he said "Pass, Shibboleth». They then 
passed up the winding staircase, consisting of three, five, seven, or more steps. [...]

After our ancient Brethren had gained the summit of the winding staircase, they arrived at the door of the middle chamber which they found open, but properly tyled against all under the Degree of a Fellowcraft by the Senior Warden, who demanded of them the Sign, Token and Word of a Fellowcraft. After they had given him those convincing proofs, he said "Pass, Shibboleth». They then passed into the middle chamber of the Temple, where they went to receive their wages... 16

Si nous revenons à la Bible, il est certain qu'elle ne suggère à aucun moment la présence de tels personnages dans le Temple ou à proximité immédiate. Le texte des Livres dits Historiques n'est même pas la relation du travail des constructeurs. Il ne présente, en effet, qu'une réflexion théologique sur l'histoire de rois d'Israël et de son peuple. Il apparait donc que tout le texte rituel n'est qu'un développement propre à la Maçonnerie. Ce premier point, élucidé par les rituels, nous conduit à un second, le parcours le long de l'escalier. La montée d'abord, qui est contrariée (opposed by) par l'opposition d'un surveillant placé à l'endroit approprié; l'entrée dans la chambre du milieu, ensuite, l'est par celle d'un autre surveillant. Tout cela n'est décodable qu'en référence au rituel. Notre attention sera ensuite attirée sur les bras tendus des personnages ( $T 603$, 08 et 09). Les rituels encore peuvent seuls expliquer ce geste: l'attente de la grippe ${ }^{17}$ de passe (pass grip), ensuite celle du signe (token), qui est une grippe différente donnée, à la suite d'une demande, comme marque prouvant sa qualité.

\subsection{Le groupe $S$}

Quant au mot de passe (pass word), le rituel en donne le sens qui élucide immédiatement le "groupe $S$ » figurant «à l'extérieur», la plante et l'eau en cascade/chute: «... a pass word, which is Shibboleth. Shibboleth... is usually depicted in our Lodges by an ear of corn near a fall of water...» ${ }^{18}$. C'est ainsi que la coprésence du cours d'eau, avec chute, et du végétal dressé sur sa tige est plus facilement explicable. Le mot appartient bien à la Bible où il est (parfois) expliqué par deux sens différents: épi de blé ou chute d'eau. Mais seul le rituel, dans son rapport intime avec les Tableaux, permet de comprendre que le végétal est un épi de blé. Le dictionnaire Webster's ${ }^{19}$ indique que le mot (Shibboleth) vient de l'hébreu et qu'il y signifie cours d'eau (stream) et qu'il était "utilisé comme mise à l'épreuve pour distinguer les Galaadites des Ephraïmites, qui prononçaient sibboleth». La référence est nettement biblique. Le texte rituel indique ensuite:

The word Shibboleth dates its origin from the time that an army of Ephraimites crossed the River Jordan in a hostile manner against Jephta, the renowned Gileaditish general. ${ }^{20}$

C'est ce qui permet de mieux comprendre l'illustration du Tb03, où la scène a été développée. Mais, ici encore, cela sous-entend des lecteurs ayant une bonne connaissance de la Bible. Le placement du "groupe $S$ » (pour Shibboleth...) n'est pas facilement compréhensible: cette scène n'a aucun lien, de près ou de loin, avec le Temple, et il n'en est donc qu'un élément extérieur. Tous les illustrateurs l'ont donc toujours placéa l'extérieur de la scène principale qui se déroule dans le Temple. L'artifice montre, par une situation décentrée, ce qui sépare une telle scène de celle du Temple. Les Tableaux illustrent graphiquement une partie de ce que les rituels énoncent. Ils ont choisi de figer l'image sur ce qui paraissait, sans doute, essentiel: les deux Surveillants, l'un avant l'escalier, l'autre devant la porte. Peu de Tableaux complètent la scène par la présence de Compagnons leur restituant les secrets. Si le parcours proposé est d'entrer dans le Temple, seul le rituel fournit des réponses claires.

\subsection{Une proposition}

La proposition des Tableaux semble être celle d'un trajet qui commence au dehors, dans le monde extérieur, où règne la nature, avec la cascade d'eau et le végétal dressé, bien visible, près de l'entrée d'un édifice. Passer à côté de cette plante n'est jamais un hasard en raison de son placement à côté de la porte. Le trajet se poursuit par la traversée de l'ouverture, l'entrée dans le bâtiment qui, par l'irradiation qu'on y a figurée, est la résidence de Dieu. On se trouve alors face à un escalier qu'on ne peut monter sans avoir été vu, ou/et contrôlé, par un premier personnage qui regarde vers l'extérieur. La première marche est largement face à l'entrée et ne suggère d'autre possibilité que de le monter, exercice qui conduit à s'élever à un deuxième niveau. Là, le parcours suggère une poursuite en ligne droite jusqu'à une deuxième entrée où se tient un deuxième personnage, qui aussi regarde à l'extérieur. On est tenté de supposer 
que le trajet continuera en passant la deuxième porte, dans un lieu qui reste caché. Les lignes du tracé, par leurs fréquences, contraignent à "monter» vers cette porte. La montée se fait en quittant le monde des lignes droites et anguleuses pour se diriger vers un monde céleste où règnent la courbe et l'arrondi, de même que la lumière omniprésente des irradiations et des êtres célestes.

Le rituel, dans ses rapports à la Bible mais aussi avec ses propres développements, permet de mieux situer le parcours suggéré par les images. Il est net maintenant qu'il ne le contrarie pas. Il semble certain, au contraire, que les Tableaux illustrent, au mieux, le contenu verbal des rituels. Le "groupe $S$ », par exemple, s'il est éclairé par le rituel et par ricochet par la Bible, ne peut pas être représenté picturalement d'une autre manière. Le rituel donne immédiatement le sens de Shibboleth, le Tableau l'illustre, sans bien entendu pouvoir «dire» le mot. Il ne peut être saisi que par la signification qu'on lui donne.

Comment dire mieux, aussi, que la présence divine règne dans le lieu qu'on représente? Les Tableaux utilisent, de manière récurrente, des symboles du divin, et les placent de telle manière qu'ils soient perçus avant tout autre élément. Cela entre dans les éléments d'une encyclopédie que toute personne au fait du religieux doit connaître, tant elle a été utilisée au cours des siècles. Comment illustrer mieux le Temple, son escalier, sa chambre du milieu? Les auteurs, les Maçons, les commentateurs de la Bible, par leur familiarité avec ce texte, ne pouvaient imaginer le Temple et ses parties autrement. Ces images représentent indiscutablement leurs convictions quant à la réalité de notions, que le poids culturel du texte sacré conduisait, de plus, à n'en pas discuter la véracité. Comment illustrer mieux un parcours ascensionnel? Les mots du rituel, transposés dans le domaine iconique, sont devenus un escalier, qui «tourne», tout en restant dans une situation spatiale visible de tous côtés. Comment, de plus, inciter le lecteur à "pénétrer" dans une chambre du milieu de meilleure manière qu'en plaçant son entrée au centre du Tableau, au point de convergence des lignes du tracé? Comment suggérer le passage du monde de la nature, où tout n'est que bruit et mouvement, au monde céleste et divin, où tout est supposé être calme et repos, silence et paix, que par la vision d'un brève portion d'un monde que l'on quitte pour le lieu où règne Dieu, lieu qu'on représentera par une bâtisse large et imposante et aux lignes régulières?
Tout cela, bien entendu, saupoudré de quelques outils rappelant que l'image s'adresse aux Maçons, héritiers du métier de construction et, à ce titre, de la science de la géométrie. Mais le rappel du métier, et de l'appartenance qu'on peut y entretenir, s'accomplit dans un ordonnancement d'éléments qui suggère que ce qui est représenté est méthodique, rationnel, géométrique, ordonné, rituel... Les Tableaux ont saisi, sur ces points, les articulations signifiantes du rituel.

\section{NOTES}

1. Le rituel de référence sera le rituel dit "anglais», dont il existe plusieurs versions assez proches.

2. J.C. Cooper, An Illustrated Encyclopaedia of Traditional Symbols, London, Thames and Hudson, 1993, p. 62.

3. Ibid., p. 54 .

4. J. Newman et G. Sivan, Le Judaïsme de A à Z. Lexique des termes et des notions de la vie juive, Paris, Biblieurope, 1987, p. 35.

5. Les références sont données avec les abréviations anglaises et françaises des Livres bibliques consultés.

6. Version du Roi Jacques.

7. IK 5:5.

8. IK 7:51.

9. La Bible. Ancien et Nouveau Testament avec les Livres Deutérocanoniques, trad. de l'hébreu et du grec en français courant, Villiers-le-Bel, Société Biblique française, 1996.

10. La Bible du Semeur, trad. en français d'après les textes originaux hébreux et grecs, avec introduction, notes et lexique, Nogent-sur-Marne, Association Biblique Internationale, 1996.

11. II Chr 4:12.

12. "Ces Colonnes étaient, en outre, ornées de deux sphères sur lesquelles étaient tracées les cartes des globes céleste et terrestre..."

13. «Pour la construction du Temple du Roi Salomon, on employa un très grand nombre d'ouvriers [...] Le salaire des Compagnons se payait en numéraire; ils allaient le recevoir dans la chambre du milieu du Temple [...]. Après que nos anciens avaient franchi le portique, ils se trouvaient au pied de l'escalier tournant, qui conduisait à la chambre du milieu ».

14. 1 Kings 6:8.

15. La Bible. Ancien et Nouveau Testament avec les Livres Deutérocanoniques, ibid.

16. «Après que nos anciens avaient franchi le portique, ils se trouvaient au pied de l'escalier tournant, qui conduisait à la chambre du milieu. Le $2^{\mathrm{e}}$ Surveillant s'opposait à leur montée et exigeait d'eux l'attouchement de passe et le mot de passe conduisant du Premier au Deuxième Grade. [...] Après que nos anciens Frères avaient donné ces preuves irrécusables au Deuxième Surveillant, il leur disait: "Passez, Schibboleth". Ils passaient et montaient l'escalier tournant qui se composait de 3, 5, 7 marches ou plus. [...] Lorsque nos anciens avaient atteint le sommet de l'escalier tournant, ils arrivaient à la porte de la chambre du milieu qu' ils trouvaient ouverte, mais 
correctement tuilée envers tout ouvrier au-dessous du Grade de Compagnon, par le Premier Surveillant, qui exigeait d'eux le signe, l'attouchement et le mot de Compagnon. Après lui avoir donné ces preuves irrécusables, celuici disait: "Passez, Shibboleth". Ils entraient alors dans la chambre du milieu du Temple, où ils allaient recevoir leur salaire...".

17. Traduit en jargon maçonnique par "attouchement».

18. «... le mot de passe est Shibboleth; qui signifie épi; et il est symbolisé ici par un épi de blé auprès d'une chute d'eau».

19. Webster's New Collegiate Dictionary, Springfield (Mass.), G. \& C. Merriam Company, 1976.

20. «Le mot Shibboleth tire son origine de l'époque où une armée des hommes d'Ephraïm traversa le Jourdain avec un comportement hostile à l'égard de Jephté, l’illustre général des hommes de Galaad [...]».

\section{SOURCES DES ILLUSTRATIONS}

Tb1 Tableau de John Cole

HAUnCH, T.O. [1962] : "Tracing Boards. Their development and their designers ", Ars Quatuor Coronatorum, London, vol. 75, p. 189 (fig. 8).

FEDDERSEN, Klaus C.F. [1982] : Die Arbeitstafel in der Freimaurerei. Bayreuth, Quatuor Coronati no 808, p. 172 (coté E/24).

MaCNulTY, W. Kirk [1991] : [Freemasonry] La Franc-Maçonnerie,

[London] Paris, [Thames and Hudson Ltd] Seuil, 1993, p.78.

Tb2 Tableau de John Browne

HaUnCH, T.O., op. cit., p. 192 (fig. 14).

FEDDERSEN, Klaus C.F., op. cit., p. 178 (coté E/30).

Tb3 Tableau de Josiah Bowring (1819)

MaCNulTY, W. Kirk, op. cit., p. 48-49.

Freimaurer. Solange die Welt besteht (catalogue d'exposition), p. 195 (coté 10/8a).

Tb4 Tableau de John Harris, "1 ${ }^{\text {re }}$ période»

FEDDERSEN, Klaus C.F., op. cit., p. 190 (coté E/42).

MACNulTY, W. Kirk, op. cit., p. 23.

Tb5 Tableau de John Harris, "1 ${ }^{\text {re }}$ période»

MaCNulTY, W. Kirk, op. cit., p. 45.

Tb6 Tableau de John Harris, "1 re période" (c1780)

Freimaurer..., op. cit., p. 199 (coté 10/13).

Tb7 Copie d'un Tableau de John Harris, "2e période»

Franc-Maçonnerie. Avenir d'une tradition (catalogue d'exposition, Musée des beaux-arts de Tours), Neuvy-le-Roi, Alfil, 1997, p. 192 (coté 6/1-2-3).

Tb8 Tableau de Harris " $2^{\mathrm{e}}$ période"

DYER, Colin F. W. [1973] : Emulation. A Ritual to Remember. Notes on the men and times in the one hundred and fifty years' history of Emulation Lodge of Improvement. 1823-1973, London, A. Lewis (Masonic Publishers), p. 208-209.

The Lectures of the Three Degrees in Craft Masonry, Addlestone, Lewis Masonic, [1974] 1994.

Tb9 Tableau de type Harris "2e période"

Rite Émulation. Deuxième grade, Paris, GLNF, 1999, p. 39.

Tb10 Tableau de Esmond Jefferies

The Logic Working of Craft Ceremonies, London, Toye, Kenning \&

Spencer, 1988, p. 92.

FEDDERSEN, Klaus C. F., op. cit., p. 249 (cotée E/99).

CARR, H. [1976] : The Freemason at Work, $7^{\text {th }}$ revised ed., Runnymede,

Lewis Masonic, 1992, p. 36. 